\title{
Multimodal system designed to reduce errors in recording and administration of drugs in anaesthesia: prospective randomised clinical evaluation
}

\author{
(c) $(1)$ (1)
}

\begin{abstract}
Alan F Merry professor ${ }^{12}$, Craig S Webster senior research fellow ${ }^{3}$, Jacqueline Hannam research coordinator $^{1}$, Simon J Mitchell associate professor ${ }^{12}$, Robert Henderson human factors specialist ${ }^{4}$, Papaarangi Reid associate professor ${ }^{5}$, Kylie-Ellen Edwards research assistant ${ }^{1}$, Anisoara Jardim research assistant ${ }^{1}$, Nick Pak research assistant ${ }^{1}$, Jeremy Cooper consultant anaesthetist ${ }^{12}$, Lara Hopley consultant anaesthetist ${ }^{12}$, Chris Frampton associate professor (statistician) ${ }^{6}$, Timothy G Short associate professor ${ }^{12}$
\end{abstract}

\footnotetext{
${ }^{1}$ Department of Anaesthesiology, Faculty of Medical and Health Sciences, University of Auckland, Private Bag 92-019, Auckland 1142, New Zealand; ${ }^{2}$ Department of Anaesthesia, Auckland City Hospital, Grafton, Auckland; ${ }^{3}$ Centre for Medical and Health Sciences Education, University of Auckland, Private Bag 92-019, Auckland 1142; ${ }^{4}$ Human Factors Group, Air New Zealand, Private Bag 92-007, Auckland 1142; ${ }^{5}$ Te Kupenga Hauora Māori, Faculty of Medical and Health Sciences, University of Auckland, Private Bag 92-019, Auckland 1142; ${ }^{6}$ Department of Medicine, Christchurch School of Medicine and Health Sciences, University of Otago, Christchurch 8140
}

\begin{abstract}
Objective To clinically evaluate a new patented multimodal system (SAFERSleep) designed to reduce errors in the recording and administration of drugs in anaesthesia.

Design Prospective randomised open label clinical trial.

Setting Five designated operating theatres in a major tertiary referral hospital.

Participants Eighty nine consenting anaesthetists managing 1075 cases in which there were 10764 drug administrations.

Intervention Use of the new system (which includes customised drug trays and purpose designed drug trolley drawers to promote a well organised anaesthetic workspace and aseptic technique; pre-filled syringes for commonly used anaesthetic drugs; large legible colour coded drug labels; a barcode reader linked to a computer, speakers, and touch screen to provide automatic auditory and visual verification of selected drugs immediately before each administration; automatic compilation of an anaesthetic record; an on-screen and audible warning if an antibiotic has not been administered within 15 minutes of the start of anaesthesia; and certain procedural rules-notably, scanning the label before each drug administration) versus conventional practice in drug administration with a manually compiled anaesthetic record.
\end{abstract} methods $(P=0.052)$. The records generated by the new system were more legible, and anaesthetists preferred the new system, particularly 
in relation to long, complex, and emergency cases. There were no differences between new and conventional systems in respect of outcomes in patients or anaesthetists' workload.

Conclusions The new system was associated with a reduction in errors in the recording and administration of drugs in anaesthesia, attributable mainly to a reduction in recording errors. Automatic compilation of the anaesthetic record increased legibility but also increased lapses in a vigilance latency task and decreased time spent watching monitors.

Trial registration Australian New Zealand Clinical Trials Registry No 12608000068369.

\section{Introduction}

Iatrogenic harm is an important public health problem with high human and financial costs. ${ }^{12}$ In anaesthesia, errors in drug administration are particularly problematic, can occasionally have devastating consequences for patients, and ought to be preventable. ${ }^{3-7}$ There have been calls for action in this regard, and in the United States the Anesthesia Patient Safety

Foundation recently held two summit meetings to address this problem. ${ }^{8-10}$ The importance of errors in drug administration in other specialties within healthcare is also well recognised. ${ }^{11-14}$

The anaesthetic record is an important clinical tool for decision making during anaesthesia and postoperatively. It is an important document for legal purposes and a source of data for research, audit, and continuous quality improvement. Inaccuracies in the recording of administered drugs can lead to subsequent errors, such as the repeated administration of a dose of drug given but not noted. This applies not only in anaesthesia but also in other settings. ${ }^{12}$ Anaesthetic records are traditionally compiled by hand, a process that accounts for about $15 \%$ of an anaesthetist's intraoperative time ${ }^{15}{ }^{16}$ Handwritten records are often incomplete and inaccurate, ${ }^{617} 18$ which might reduce or negate their value for any of the above purposes.

To enhance safety and quality through system improvement, a set of strategies designed to reduce errors in the administration and recording of drugs given during anaesthesia and facilitate and improve the quality of anaesthetic record keeping has been incorporated into a novel system (SAFERSleep: Safer Sleep LLC, Nashville, TN). ${ }^{19-21}$ The new system is in use in several hospitals in New Zealand and the United Kingdom and, in particular, has been used for most anaesthetics at Auckland City Hospital since mid-2005. Certain aspects of the system have been evaluated in other settings-for example, several studies have shown that the use of bar coding improves drug safety. ${ }^{12-14} 22{ }^{23}$ There have also been numerous studies of electronic record keeping in anaesthesia and in healthcare generally. The value of electronic health records, however, has not been fully elucidated, and few relevant studies have used a prospective randomised design. ${ }^{24-26}$

Many apparently effective safety innovations have been introduced in medicine in an attempt to reduce error or improve quality. Most innovations of this type come at some cost, and the new system is no exception. ${ }^{21}$ As a minimum this cost represents an opportunity loss in respect of other possible uses of limited healthcare resources. ${ }^{27}$ More worryingly, unintended (and perhaps undesirable) consequences are also a possibility with any new process. ${ }^{28}{ }^{29} \mathrm{We}$ conducted a prospective randomised open label clinical trial to evaluate the new system's impact on errors in the recording and administration of drugs in anaesthesia and on anaesthetists' vigilance and workload.

\section{Methods \\ Hypotheses}

Our primary (null) hypothesis was that there would be no significant difference between the new system and conventional methods in the rate of errors in the recording and administration of medications or in the number of lapses in responding to a vigilance latency test. We also hypothesised that there would be no differences in compliance with the need to administer prophylactic antibiotics within one hour of incision, in outcomes in patients, in anaesthetists' workload (either self recorded or recorded by observers), in the preferences of participating anaesthetists, or in the legibility of the anaesthetic records. Finally, we hypothesised that there would be no difference between Māori and other patients in our primary outcome variables or in anaesthetists' workload.

\section{Trial design and participants}

The study protocol and registration details were updated twice before the finalisation of data entry and subsequent analysis of results to add the terms "iv" and "iv drug" for clarification and because it became apparent that components of the combined primary outcome variable, as initially defined, had different denominators and could not be added to each other. Data were collected from five designated operating theatres in the adult anaesthetic departments of Auckland City Hospital. Participating anaesthetists were familiarised with the trial and its objectives and gave written informed consent before taking part. All participating anaesthetists had received formal training in the use of the new system before the start of this study. Before surgery, all patients scheduled for anaesthesia in the designated study theatres were given an information sheet that informed them of the nature of the study and the consequent presence of an observer during their surgery and that they could decline to participate in the study. For clarity, we have used the term "case" throughout to mean one patient undergoing anaesthesia for surgery on one occasion.

The study's statistician (CF) performed randomisation by week, with treatment allocation codes in blocks of four and with stratification for study theatre, with a computer generated random sequence (Microsoft Excel, Redmond, WA). Theatres were set up for provision of anaesthesia with either the new system or conventional methods according to the randomisation schedule at the start of each week and remained so for that week. The operating theatres selected and surgical specialties represented were chosen to give a broad case mix. No attempt was made to manipulate the scheduling of anaesthetists to study theatres. Observers attempted to contact anaesthetists the day before each study day to alert them to the study and (if they asked) the system under which they would be working. Anaesthetists could decline to participate on any given day without retracting their overall consent.

\section{Interventions}

The new system has previously been described in detail. ${ }^{20}$ It includes the following elements:

- Customised drug trays designed to promote a well organised anaesthetic workspace and aseptic technique

- Pre-filled syringes for the most commonly used anaesthetic drugs (see table A in appendix on bmj.com for details)

- Large legible drug labels, colour coded according to standards adopted in several countries ${ }^{30-33}$ 
- A barcode reader linked to a computer, speakers, and touch screen to provide automatic auditory and visual verification of the selected drug immediately before each administration and to record its administration (keyboard entry of drug name is possible as an alternative)

- An on-screen visual and auditory warning if an antibiotic has not been administered within 15 minutes of the start of anaesthesia

- Purpose designed drug trolley drawers

- Automatic compilation of an anaesthetic record, on screen in real time and as a paper print-out at the end of anaesthesia.

The following six procedural rules are followed for the correct use of the system:

- Appropriate organisation of syringes and ampoules

- Ampoules appropriately laid out at the start of the case

- All empty ampoules and syringes retained for reconciliation

- All syringes labelled

- Computerised voice prompt audible throughout each anaesthetic

- Medication label scanned before each drug administration.

Of these, the last two principles are considered the most crucial to the effectiveness of the new system.

The conventional management option included the following elements:

- A standard drug tray to hold the syringes and ampoules

- A standard fully stocked drug trolley

- All drugs drawn up by the anaesthetist

- Small standardised colour coded drug labels, to be applied by the anaesthetists

- Standard anaesthetic record chart to be filled in by hand, with usual access to data routinely logged by the anaesthetic monitor if desired.

\section{Data collection}

Trained observers collected data in the operating theatre. Training included reading an introductory textbook of anaesthesia, ${ }^{34}$ observing at least five cases, an emphasis on discreet observation with minimal unnecessary conversation, and collecting data simultaneously with an experienced trained observer for a minimum of 10 cases or until acceptable concordance was achieved. An investigator experienced in observational research $(\mathrm{RH})$ judged competence to collect actual study data independently. For the first 416 cases the observers were present throughout to collect workload and task analysis data. For the remainder they were present for most, but not necessarily all, of each case. Observers were, however, present in the operating theatre at the beginning and end of every case. Two investigators (RH and PR) with no relevant conflict of interest were explicitly asked to oversee the study processes; among other things they made several visits to the study theatres to personally inspect the processes of observation and data collection. Two overseas collaborators with no relevant conflicts of interest visited Auckland City Hospital during the study to inspect the data collection processes (again by visiting the operating theatres) and other aspects of the study.

\section{Recording and administration of drugs (reconciliation}

process)_To facilitate identification of errors in the recording and administration of drugs, we took a full inventory of the contents of the anaesthetic drug drawers before each case. Anaesthetists were asked to retain all syringes and vials and to avoid discarding unused contents of syringes. Empty sharps bins were used to facilitate counting of any drugs inadvertently discarded during the case. At the end of each case, the drugs used and the total doses given were identified through these means and through reconciliation of the remaining contents of the drug drawers against the preoperative inventory. These drug names and total doses were compared with the drug administrations recorded on the final anaesthetic record and any discrepancies noted.

Vigilance-We used a vigilance latency task to assess anaesthetists' vigilance and spare work capacity. ${ }^{35}$ This task required the anaesthetists to acknowledge the illumination of an easily visible bright light on the anaesthetic machine, which appeared at random intervals of nine to 14 minutes. If it was not acknowledged within 300 seconds, the light went out and the failure to respond was classed as a lapse of vigilance. Vigilance data were recorded with customised software operating on a personal digital assistant (Hewlett Packard iPAQ, Houston, TX). The vigilance latency task was manually activated when the anaesthetist entered the operating theatre at the start of each case and continued until the anaesthetist left with the patient.

Task analysis and workload assessment-All tasks performed by anaesthetists and anaesthetic trainees in the operating theatre were directly coded and entered on to a computer with a custom written task analysis program, based on the work of Weinger ${ }^{36} 35$ but modified to focus on tasks specifically relevant to drug administration and anaesthetic record making. The program calculated the duration of defined tasks from start and stop times entered via touch screen by the observers. Additional free text descriptions and comments were possible. Observation began when the anaesthetist(s) entered the operating theatre at the start of each case, included preparation before entry of the patient, and continued until departure of the patient at the end of the case. At random intervals of between seven and 15 minutes, the computer program prompted recordings of perceived workload with the Borg workload score. ${ }^{37}$ This score is a 15 point interval scale between six and 20, where 12 is the workload of a normal tracheal intubation. Workload scores were assessed independently by the observing researcher and self reported by the anaesthetist.

Legibility -As a measure of legibility we examined the first and last 100 records in each arm of the study and recorded the total time to extract the number of discrete blood pressure and temperature measurements recorded; the number of intravenous drugs recorded; and the number of bolus doses of medication recorded. $^{38}$

Compliance-At the end of each case the observer assessed the anaesthetists' compliance with the procedural rules as described above for the correct use of the new system. Each item was scored as follows: $y e s=2$, sometimes $=1, n o=0$. The scores were summed and the result expressed as a percentage of the maximum possible score.

Ratings-Anaesthetists who managed 10 or more study cases were asked to rate the new system and conventional methods in respect of 18 attributes and six case types with $100 \mathrm{~mm}$ unmarked visual analogue scales with appropriate anchors (indicating dissatisfaction at $0 \mathrm{~mm}$ and maximum satisfaction at $100 \mathrm{~mm}$ ).

Ethnicity-Ethnicity of patients was recorded. 


\section{Outcomes}

\section{Primary outcomes}

The primary outcome variable was the composite of errors in recording or administering intravenous drugs. Errors in drug administration included giving the incorrect drug (substitution) and failing to give the intended drug (omission). Errors in drug recording included failing to record an administered drug; recording an administered drug name but failing to record the dose; and discrepancy between the recorded total dose and the dose calculated through the reconciliation process by observers. A panel of four anaesthetists blinded to the treatment arm in which the discrepancies occurred evaluated dose discrepancies. For each drug, consensus was reached on the minimum discrepancy that would be clinically relevant in the context of the dose typically used for each drug in Auckland City Hospital and of the pharmacology of the drug (see table B in appendix on bmj.com). A discrepancy in dose was deemed clinically relevant, and therefore counted, when the total dose documented by the anaesthetist on the final anaesthetic record differed from that documented by the observer by this amount or more. In cases where there was unresolved inconsistency between the identities of the reconciled ampoule, the labelled syringe, and the drug recorded by the anaesthetist the error was classified as "incorrect label." The composite primary outcome variable and the two individual error types were quantified as the number of errors per administration for each case and represented as overall rates per 100 administrations.

\section{Secondary outcomes}

Patient outcomes-We reviewed the hospital records of all patients after their discharge and recorded the following predefined adverse outcomes: anaphylaxis, myocardial infarction, sepsis, septic shock, or death. We also sought any evidence of harm attributable to a drug error. In addition, for each case we determined whether antibiotics would normally be required as a matter of surgical policy at the hospital and, if so, whether they were administered within the 60 minutes before surgical incision.

System outcomes-Task analysis and workload assessments, legibility of anaesthetic records, compliance with procedural rules of the new system, and questionnaire ratings were analysed as described below.

\section{Statistics}

The study statistician (CF) analysed the data with SPSS version 15.0 (SPSS, Chicago, IL). A subset of data from $2.5 \%$ of the cases, selected randomly, was re-entered to check the accuracy of the data entry. A two tailed probability of $\mathrm{P} \leq 0.05$ was predefined as indicative of significance. The primary null hypothesis - that there would be no significant difference between the new system and conventional methods in errors in recording or errors in administration of drugs - was tested with a general linear mixed model that included "operating theatre week" as a random effect. The number of errors per administration was calculated for each case. This involved summing all the relevant errors for the case and dividing by the total number of administrations for that case. The unit of analysis was the case and the unit of randomisation was the operating theatre week. The results are reported as errors per 100 drug administrations. The effects of patients' American Society of Anesthesiologists (ASA) score, type of anaesthetic procedure (emergency/elective), ethnicity, sex, and age on error rates were also assessed with a general linear mixed model that simultaneously tested all these effects.
We compared the proportion of anaesthetists experiencing lapses in vigilance between randomised groups using $\chi^{2}$ tests or Fisher's exact tests when the expected error rates were low. Total times spent on individual and collective tasks, as recorded by observers, and the mean workload assessments were also compared between randomised groups with general mixed linear models that included "operating theatre week" as a random effect. The appropriateness of these parametric models for testing the primary and secondary outcomes was confirmed with normality plots of model residuals. Spearman's rank correlation coefficients were used to assess the strength of the relations between the error rate and anaesthetist's self reported workload, and the error rate and compliance scores for use of the new system, per case. Workload scores as assessed by the observer were correlated with anaesthetists' self reported workload scores with Spearman's correlation coefficients. The total time spent extracting information as an assessment of legibility of the anaesthetic record was compared between randomised groups with an independent $t$ test.

We calculated mean visual analogue scale ratings for the new system and for conventional methods for each anaesthetist over the 18 attributes and the six scores relating to case types (that is, a mean of the 24 items) and compared them between randomised groups with a paired $t$ test. Differences in individual items were evaluated post hoc (given significance in the overall comparison) with paired $t$ tests with a Bonferroni correction to allow for multiple testing.

\section{Sample size estimation}

No comparable data were available for observational research on errors in recording or administering drugs in the clinical setting, though facilitated incident recording has identified one error in every 133 cases and an average of about 10 drug administrations per case. ${ }^{36}$ In observational research with a human patient simulator, the composite error rate for recording and administration of drugs was about 0.3 per administration, ${ }^{6}$ but this study was designed to greatly increase the likelihood of error. If one assumed one error per 10 administrations in the control group, about 3300 administrations (that is, about 330 cases) per group would be needed to show a $20 \%$ reduction with $80 \%$ power and a two tailed $\alpha=0.05$. This calculation is based on the binomial distribution of the number of errors per case, allowing for 10 administrations per case and then using this to estimate variation between cases in the error rate per administration. Given the lack of clear data to support these assumptions, and taking into account the pragmatic limitations of our study and uncertainty over the assumption of independence inherent in this estimate, we aimed to study at least 500 cases in each study arm. Because of the intensive nature of the task analysis data collection and the greater sensitivity of these continuous data, the collection of this information was ultimately limited to about the first 400 cases.

\section{Results}

Data were collected from five designated operating theatres in Auckland City Hospital between March 2008 and February 2009 , at which time recruitment targets were met. A total of 1748 cases were managed in the study theatres during the time of the trial; two were excluded because the patients indicated a preference not to participate and 502 because no observer was available; 613 of the eligible cases were allocated to the new system and 631 to conventional methods. On the day, anaesthetists declined to participate in 46 (new system) and 111 (conventional) of these study cases respectively $(\mathrm{P}<0.001)$. One 
record made by the new system was lost because of a computer malfunction and 11 made by conventional methods could not be retrieved from the hospital's record system so data were analysed from 1075 cases (566 with the new system and 509 with conventional methods, figure $\Downarrow$ ). There was $100 \%$ concordance between the records in the primary dataset and the $2.5 \%$ of records re-entered for quality control.

\section{Anaesthetists, patients, and cases}

Eighty nine anaesthetists took part in the study, including 54 specialists and 35 trainees. The specialists had a median of 10 years' (range 1-35) experience. Nine anaesthetists refused to participate at all, and five withdrew completely after limited participation. Some anaesthetists refused to participate on particular days; reasons for this included not wanting to use the conventional manual record for complicated patients (none cited not wanting to use the new system as a reason), reluctance to deal with the study while teaching medical students, finding participation onerous, and disliking taking part in the study.

Characteristics of patients and the mix of surgical specialties, emergency/elective cases, ASA scores, and duration of anaesthesia were well balanced between the two groups (table $1 \Downarrow)$.

The total number of drug administrations with the new system and conventional methods was 5680 and 5084, respectively, equating to an average of about 10 drug administrations per case for both.

\section{Main outcomes \\ Errors}

There were 471 errors in drug administration or recording from 5680 administrations in 566 cases with the new system and 488 errors from 5084 administrations in 509 cases with conventional methods. The mean rate of these errors per 100 administrations, calculated from the general linear mixed model, was 9.1 (95\% confidence interval 6.9 to 11.4 ) with the new system (one in 11 administrations) and 11.6 (9.3 to 13.9) with conventional methods (one in nine administrations) $(\mathrm{P}=0.045)$, a reduction in the error rate of 2.5 per 100 administrations ( 0.3 to 4.8$)$ or $21 \%$ (table $2 \Downarrow$ ). The rate of errors in drug administration per 100 administrations was 0.16 with the new system (one in 625) and 0.33 with conventional methods (one in $303 ; \mathrm{P}=0.120$ ). The rate of recording error per 100 administrations was 9.68 with the new system (one in 10) and 12.10 with conventional methods (one in eight; $\mathrm{P}=0.049$ ).

Errors in drug recording and administration were more common in male than in female patients, but there were no significant differences between operating theatres, ASA scores, elective/emergency cases, or ethnic groups (table $3 \Downarrow$ ), and error rate was not related to patient's age. When indicated, antibiotic prophylaxis was given before incision in $94 \%$ of patients with the new system and $93 \%$ with conventional methods $(\mathrm{P}=0.48)$.

\section{Vigilance}

Evaluable data were obtained for 944 cases. Lapses in response to the vigilance latency task occurred in $12 \%(58 / 471)$ of cases with the new system and 9\% (40/473) with conventional methods $\left(\mathrm{P}=0.052, \chi^{2}\right.$ test $)$.

\section{Secondary outcomes Patients' outcomes}

No errors identified in this study caused any harm to any patient. Nine patients in each group experienced a major complication (anaphylaxis, myocardial infarction, sepsis or septic shock). Five patients died during their hospital admission in the group anaesthetised with the new system compared with six in the conventional management group.

\section{Task analysis and workload assessment}

Task analysis and workload assessment data were collected for 416 cases. Less time was spent record keeping with the new system than with conventional methods and slightly less time observing monitors; on the other hand more time was spent observing patients and preparing and administering drugs (table $4 \Downarrow)$.

The mean self reported Borg workload scores for the two systems were identical, at 13.2 (12.8 to 13.6). Patients' ethnicity had no effect on anaesthetists' workload. There was a strong correlation between these self reported scores and the observer ratings $(r=0.76, \mathrm{P}<0.001)$, but anaesthetists' ratings were 5\% higher than the observers'. The error rate per drug administration correlated with the anaesthetists' mean rating of workload for each case $\left(r_{s}=0.15, \mathrm{P}=0.004\right)$.

\section{Legibility}

It took a mean time of $2 \mathrm{~min} 18 \mathrm{sec}$ (SE $4 \mathrm{sec}$ ) to extract the three specified pieces of information from each record made with the new system and $3 \mathrm{~min} 41 \mathrm{sec}(8 \mathrm{sec})$ from each record made by the conventional system $(\mathrm{P}<0.001)$.

\section{Compliance with principles of the new system}

The mean compliance score for cases in which the new system was used was $81 \%$ (SE $0.8 \%$, range $40-100 \%$ ). The proportion of cases (from 456 with evaluable ratings) in which anaesthetists complied with individual principles of the new system were $78 \%$ for appropriate organisation of syringes and ampoules; $89 \%$ for ampoules appropriately laid out at the start of the case; $92 \%$ for all empty ampoules and syringes retained for reconciliation; $84 \%$ for all syringes labelled; $79 \%$ for computerised voice prompt audible throughout the case; and $62 \%$ for medication label scanned before each drug administration.

Anaesthetists were fully compliant with all the principles of the new system in only 83 of 456 cases (18\%). Compliance score was negatively correlated with error rate $(r=-0.11, \mathrm{P}=0.016)$, with $42 \%$ of the variation among compliance scores attributable to differences between anaesthetists. The mean error rate per 100 administrations was 6.0 (3.1 to 8.8) when the anaesthetist complied with the two key principles of scanning the drug barcode before administering each drug and keeping the voice prompt active, and 9.7 (8.4 to 11.1) when the anaesthetist did not comply with them $(\mathrm{P}=0.004)$.

\section{Rating scales}

Twenty eight anaesthetists participated in 10 or more study cases; of these $21(75 \%)$ completed a rating sheet for both the new system and conventional methods. The mean rating for all questions was $74 \mathrm{~mm}$ (SE $2 \mathrm{~mm}$ ) for the new system and 60 $\mathrm{mm}(3 \mathrm{~mm})$ for conventional methods; the mean difference was $14 \mathrm{~mm}$ ( $5 \mathrm{~mm}$ to $22 \mathrm{~mm}$; $\mathrm{P}=0.003)$. After Bonferroni correction, there were significant differences in ratings, all favouring the new system, in the following scales: overall satisfaction 
( $\mathrm{P}<0.01)$, effort required $(\mathrm{P}=0.02)$, usefulness $(\mathrm{P}=0.05)$, and completeness $(\mathrm{P}=0.01)$ of the anaesthetic record generated by this method and attitude to use of the system for all cases $(\mathrm{P}=0.002)$. Differences in ratings for reliability of the system, its inherent safety as an approach to anaesthesia, time available for monitoring patients, attitude to advocating use in another institution, and the usefulness and safety of the drug trays, syringe labelling, methods for checking drug identity, and design of the drug trolley did not reach significance. The new system was preferred over conventional methods for long $(\mathrm{P}<0.01)$, complex $(\mathrm{P}<0.01)$, and emergency $(\mathrm{P}=0.02)$ cases; differences in ratings for short, simple, and routine cases were not significant.

\section{Discussion}

In comparison with conventional methods, use of a new system designed to reduce errors in anaesthesia was associated with a $21 \%$ reduction in the rate of errors in the recording and administration of drugs, more legible anaesthetic records that were compiled in half the time, and more time devoted to observing patients. On the other hand, less time was spent observing monitors, and there were more lapses in a vigilance latency task. Evaluations indicated an overall preference for the new system over conventional methods but emphasised the reduction in effort required and the completeness and usefulness of the records rather than the safety aspects of the system. This preference was also reflected by the number of cases in which anaesthetists declined to participate in the study on the day: 46 with the new system compared with 111 with conventional methods $(\mathrm{P}<0.001)$. Preferences for the new system were most evident for long, complex, and emergency cases. Slightly more time was spent drawing up and administering drugs with the new system. There was no difference in patients' outcome, and no harm was caused by any of the errors observed in this study. No difference in self rated workload was seen between study arms.

There is international evidence of substantial ethnic disparities in the quality of healthcare ${ }^{39}$ and some evidence that such disparities might also apply to iatrogenic harm. ${ }^{40} \mathrm{We}$ therefore took the opportunity to explore the possibility of ethnic disparity in the delivery of anaesthesia with a view to perhaps designing a future study with the primary aim of investigating this issue. In fact we found no evidence of any difference between the main ethnic groupings evaluated.

Our findings were broadly consistent with previous evaluations of the new system. Earlier studies found that drug preparation times were reduced but that participants had access to a greater number of prefilled syringes. ${ }^{21}{ }^{23}$ Our rate of errors in drug administration was higher than previous estimates by our group using facilitated incident reporting ${ }^{322}$ and other studies that used standard incident reporting. ${ }^{41}$ This finding suggests that many anaesthetic errors remain unidentified by the person who makes them and that incident reporting tends to underestimate the problem.

\section{Clinical importance of findings}

Errors in drug administration are an ongoing source of concern in anaesthesia ${ }^{89}$ and in healthcare generally. ${ }^{12}$ The difference in administration errors was not significant, and no harm was identified from any of our errors, but we were not able to power our study for these end points. Our group has previously shown a significant reduction in drug administration error in incident reports from cases using the new system compared with those using conventional methods in over 74000 anaesthetics. ${ }^{22}$ The potential for harm from drug administration error is substantial, ${ }^{45}$ though some are more hazardous than others-for example, the risk of substituting a drug for one of the same class (such as one opioid for another) is likely to be lower than the risk of substituting a drug for one of a different class, and the consequences of mislabelling or omitting a drug depends on the drug involved (table 5) $\Downarrow$. Recording errors, although perhaps less likely to cause immediate harm to a patient, also have potential to impact on care particularly in the post-anaesthetic care unit and when patients are handed over from one anaesthetist to another. We have included only those dose discrepancy errors evaluated as clinically important, and there were many more that were discarded as trivial but that, nevertheless, were undesirable. It might be thought self evident that electronic records would be more accurate than manual ones, ${ }^{25}{ }^{42}$ but the recording errors analysed in our study involved information that had to be entered interactively, so this was not a foregone conclusion. ${ }^{25} 42$

The higher number of lapses in the vigilance latency task with the new system was unexpected. Using a similar test, Weinger and colleagues showed no significant difference in vigilance latency between groups keeping manual or electronic records and concluded that electronic record keeping did not affect workload or vigilance. ${ }^{43}$ Our study is much larger, and we evaluated lapses because we thought that a failure to respond for five minutes or more would matter if the missed information were clinically relevant. Anecdotally, there was widespread scepticism among participants over the clinical relevance of the vigilance latency task, but impact on vigilance is a controversial aspect of electronic record keeping and our finding suggests that further investigation of this potentially negative effect is warranted. Ideally, future work should evaluate the times to respond to important changes in patients' physiological status.

Our findings in relation to compliance with the systems' principles are of interest. There was no difficulty getting anaesthetists to use the recording aspects of the system and to comply with some of its safety principles (the mean compliance score was $81 \%$ ) but, despite ongoing efforts to provide education and motivation in this regard, full compliance with these principles occurred in only $18 \%$ of cases. Notably, this subset of participants, who engaged strongly in using the system as it was designed to be used, made significantly fewer errors. Evaluative ratings emphasised other perceived advantages of the system rather than its potential contribution to safety. Difficulty has also been found in engaging clinicians in other safety initiatives, notably in relation to hand washing. ${ }^{44}$ This might reflect complacency about the problem of iatrogenic harm in healthcare in general or about errors in drug administration in particular or perhaps scepticism about the principles of the system. We think the data on the importance of drug administration error in anaesthesia are compelling ${ }^{3945}$ and hope that the negative correlation between compliance with the principles and the rate of error seen in this study will encourage greater compliance in future.

\section{Weaknesses of the study}

The study was carried out in a single centre in which the system had been in use for five years, with a sample of convenience; this might limit the degree to which its findings apply in general (that is, its external validity). There might have been various observer effects, but these would presumably have applied to both groups ${ }^{46}{ }^{47}$ We could not blind the study. In fact, even concealment proved impractical as several participants indicated early in the study that they preferred to receive forewarning of the method they were to use on any particular day. Nine 
anaesthetists declined to participate in the study at the outset, and five more withdrew early in the study (14\%). On the day, anaesthetists declined to participate in 46 cases randomised to the new system and 111 cases randomised to conventional methods (13\%). Anaesthetists were less likely to consent to taking part in the study when anaesthetising complex cases, when there was a preference for using the new system. This lack of equipoise in attitudes to the new system was a potential source of bias. Because of the occasional unavailability of our observers, we were unable to collect data on all cases. This too could have introduced bias, although it involved no apparent systematic element. Because of limited resources we were able to collect data only from scheduled operating lists, involving predominantly elective cases during working hours, so no inference is possible in relation to emergency cases done out of hours. We had difficulty categorising five errors in which the label on the syringe referred to one drug (such as vecuronium), the empty ampoule contained another (such as atracurium), and the drug recorded was the one in the ampoule (in this case atracurium). Some might argue that these events were not errors, but they reflect poor practice, and there was no objective way of being sure what the anaesthetist actually intended in each case. These events were evenly distributed (that is, 2:3) between the two arms of the study so have little potential to bias our results. In those that occurred during use of the new system the barcode scanning check was probably not used. Imperfect compliance with the system's principles might also have reduced the effect size seen in our primary outcome variable from that potentially achievable with its correct use. The study was too small to investigate administration errors or harm but is by far the largest of its type (that is, randomised prospective investigation of the problem of drug administration error with data collection by observers) investigating this problem that we know of. It is not clear why errors were more common in male than in female patients, but study groups were well balanced in this regard so this difference is unlikely to have biased our results. The conflict of interest of some investigators was a potential source of bias. This risk was explicitly addressed by the inclusion of senior co-investigators with no conflicts of interest and by asking independent overseas collaborators to visit and review the study processes. In addition, none of the investigators with conflicts of interest were involved in the analysis of the data and the study was registered and independently funded.

\section{Future research}

Our results suggest room for improvement in the accuracy of drug recording and administration in anaesthetic practice, whether with the new system or with conventional methods. Our findings also provide objective evidence for the efficacy of the principles and rules underlying the system as part of the ongoing iterative process of its continuous improvement. Many of these are not protected but simply a matter of good practice (even checking before administration could be carried out with a second person instead of the computer and barcode reader ${ }^{48}$ ), so they could be applied immediately by anyone. Extension of these principles to drug administration in other areas of healthcare would be worth exploring. Qualitative research into the human and design factors that influence compliance with the system's safety principles could be worthwhile. It might also be worth investigating the effectiveness of role models and of various educational and motivational approaches in this regard, particularly in relation to doctors in training.

\section{Conclusions}

We have added to previous evidence 20232248 that supports the efficacy and clinical use of a multimodal approach to improving drug administration and record keeping in anaesthesia, which includes the use of barcodes and computers in checking the identification of drugs before administration. We have shown that this approach reduces a composite end point of errors in the recording and administration of drugs, increases the legibility and reduces the time spent creating anaesthetic records, and increases time spent observing patients during anaesthesia. Conversely, it decreases time spent watching monitors and increases lapses in a vigilance latency task. Compliance with the systems' principles evaluated in this study was inversely related to error rates, but achieving high levels of compliance was difficult. This study adds to the evidence informing effective system redesign for drug administration and record keeping in anaesthesia, and potentially other areas of healthcare as well. It is a step forward in an ongoing process of continuous quality improvement in anaesthesia.

We thank the anaesthetists who participated in this study, the anaesthetic technicians and theatre nurses who facilitated it, and the following individuals for their contributions: Ravi Mahajan and Joan Russell (for visiting Auckland to verify study processes), Steven Duffey, Martijn Hagen, and Joost Verhelst (for data collection), Matt Weinger (for access to information on task analysis), Johan van Schalkwyk (for advice and for commentary on the manuscript), and David Merry (for writing the task analysis and vigilance software).

Contributors: AFM conceived this study and is guarantor. AFM, CSW, TGS, JC, and PR designed and initiated the study. JH, AJ, K-EE, and NP were responsible for recruitment of anaesthetists and patients and data recording, JH and K-EE prepared the database, and CF was responsible for statistical analysis. $\mathrm{RH}$ and $\mathrm{PR}$ monitored processes, with particular reference to managing conflicts of interest. TGS wrote the first draft of the manuscript, AFM, JH, and SJM revised the manuscript, and SJM coordinated final preparation. JC, LH, AFM, and SJM provided oversight and support in the clinical locations. All authors contributed to and approved the final version of the paper, had full access to all of the data, and can take responsibility for its integrity.

Funding: This project was supported by grant 07/269R from the Health Research Council of New Zealand and a supplementary grant from the Green Lane Research and Educational Fund. These funders were not involved in the study design; in collection, analysis and interpretation of data; in writing the report; or in the decision to submit the article for publication.

Competing interests: All authors have completed the ICMJE uniform disclosure form at www.icmje.org/coi_disclosure.pdf and declare that AFM, TGS, JC, and CSW are shareholders in Safer Sleep. AFM is a director of Safer Sleep, holds about $9 \%$ of its shares, and advises the company on the design of its products. TGS, JC, and CSW are minor shareholders. The intellectual property of the new system is owned by Safer Sleep, although some patents are in the name of AFM (as inventor). AFM and CSW have been authors on several previous publications evaluating the new system.

Ethical approval: This study was approved by the Northern $\mathrm{Y}$ regional ethics committee and New Zealand health and disability ethics committee, New Zealand (No NTY/07/10/112).

Data sharing: No additional data available.

Department of Health. An organisation with a memory-report of an expert group on learning from adverse events in the NHS. Stationery Office, 2000.

2 Institute of Medicine. To err is human: building a safer health system. National Academy Press, 2000.

3 Webster CS, Merry AF, Larsson L, McGrath KA, Weller J. The frequency and nature of drug administration error during anaesthesia. Anaesth Intensive Care 2001;29:494-500. 


\section{What is already known on this topic}

Errors in the recording and administration of drugs are common in anaesthesia specifically and healthcare generally and can lead to poor care and harm patients

Checks with barcodes and computers before administration of drugs are feasible and can reduce such errors, although there are few randomised trials to show this

\section{What this study adds}

In a prospective randomised controlled trial we showed that a multimodal approach decreased errors in recording or administering drugs, reduced the time spent in keeping records, and improved their legibility; more time was spent watching patients

There were more lapses in a vigilance latency task and less time was spent watching monitors

Compliance with principles of safe drug administration was inversely associated with error rates, but achieving high levels of compliance with the safety principles of drug administration was difficult

4 Abeysekera A, Bergman IJ, Kluger MT, Short TG. Drug error in anaesthetic practice: a review of 896 reports from the Australian incident monitoring study database. Anaesthesia 2005;60:220-7.

5 Merry AF, Peck DJ. Anaesthetists, errors in drug administration and the law. $N Z$ Med J 1995;108:185-7.

6 Merry AF, Weller JM, Robinson BJ, Warman GR, Davies E, Shaw J, et al. A simulation design for research evaluating safety innovations in anaesthesia. Anaesthesia 2008;63:1349-57.

7 Webster CS. The iatrogenic-harm cost equation and new technology. Anaesthesia 2005;60:843-6.

8 Orser BA, Byrick R. Anesthesia-related medication error: time to take action. Can J Anaesth 2004;51:756-60.

9 Merry AF, Webster CS. Medication error in New Zealand-time to act. NZ Med J 2008;121:6-9.

10 Eichhorn J. APSF hosts medication safety conference: consensus group defines challenges and opportunities for improved practice. APSF Newsletter 2010;25:1-7.

11 Stewart M, Purdy J, Kennedy N, Burns A. An interprofessional approach to improving paediatric medication safety. BMC Med Educ 2010;10:19.

12 Poon EG, Keohane CA, Yoon CS, Ditmore M, Bane A, Levtzion-Korach O, et al. Effect of bar-code technology on the safety of medication administration. $N$ Engl J Med 2010;362:1698-707.

13 DeYoung JL, Vanderkooi ME, Barletta JF. Effect of bar-code-assisted medication administration on medication error rates in an adult medical intensive care unit. $A m \mathrm{~J}$ Health Syst Pharm 2009;66:1110-5.

14 Helmons PJ, Wargel LN, Daniels CE. Effect of bar-code-assisted medication administration on medication administration errors and accuracy in multiple patient care areas. Am J Health Syst Pharm 2009;66:1202-10.

15 Kennedy PJ, Feingold A, Wiener EL, Hosek RS. Analysis of tasks and human factors in anesthesia for coronary-artery bypass. Anesth Analg 1976;55:374-7.

16 McDonald JS, Dzwonczyk RR. A time and motion study of the anaesthetist's intraoperative time. Br J Anaesth 1988;61:738-42.

17 Gibbs RF. The present and future medicolegal importance of record keeping in anesthesia and intensive care: the case for automation. J Clin Monit 1989:5:251-5.

18 Rowe L, Galletly DC, Henderson RS. Accuracy of text entries within a manually compiled anaesthetic record. Br J Anaesth 1992;68:381-7.

19 Leape LL, Berwick DM. Five years after to err is human: what have we learned? JAMA 2005;293:2384-90.

20 Merry AF, Webster CS, Mathew DJ. A new, safety-oriented, integrated drug administration and automated anesthesia record system. Anesth Analg 2001:93:385-90.

21 Webster CS, Merry AF, Gander PH, Mann NK. A prospective, randomised clinical evaluation of a new safety-orientated injectable drug administration system in comparison with conventional methods. Anaesthesia 2004;59:80-7

22 Webster CS, Larsson L, Frampton CM, Weller J, McKenzie A, Cumin D, et al. Clinical assessment of a new anaesthetic drug administration system: a prospective, controlled, longitudinal incident monitoring study. Anaesthesia 2010;65:490-9.

23 Merry AF, Webster CS, Weller J, Henderson S, Robinson B. Evaluation in an anaesthetic simulator of a prototype of a new drug administration system designed to reduce error. Anaesthesia 2002; 57:256-63.

24 Block FE Jr. Automatic anesthesia record keeping. J Clin Monit 1989;5:284-6.

25 Reich DL, Wood RK Jr., Mattar R, Krol M, Adams DC, Hossain S, et al. Arterial blood pressure and heart rate discrepancies between handwritten and computerized anesthesia records. Anesth Analg 2000;91:612-6.

26 Schiff GD, Bates DW. Can electronic clinical documentation help prevent diagnostic errors? N Engl J Med 2010;362:1066-9.

27 Runciman B, Merry A, Walton M. Safety and ethics in healthcare: a guide to getting it right. Ashgate, 2007.

28 Tenner EW. Why things bite back-technology and the revenge of unintended consequences. Vintage Books, 1997.
29 Weinger MB. The impact of health care reform on anesthesiologists and their patients in the United States: an ergonomic perspective. Acta Anaesthesiol Sin 1996;34:27-32.

30 Standard specification for user applied drug labels in anesthesiology (D4774-94). American Society for Testing and Materials, 1995.

31 User-applied labels for use on syringes containing drugs used during anaesthesia (AS/NZS 4375:1996). Standards New Zealand, 1996.

32 Standard for user-applied drug labels in anaesthesia and critical care (Z264.3-98). Canadian Standards Association, 1998.

33 Anaesthetic and respiratory equipment. User-applied labels for syringes containing drugs used during anaesthesia. Colours, design and performance (ISO 26825:2008). International Organization for Standardization, 2008.

34 Dripps RD, Eckenhoff JE, Vandam LD, Longnecker DE, Murphy FL. Introduction to anesthesia. 9th ed. Saunders, 1997.

35 Weinger MB, Reddy SB, Slagle JM. Multiple measures of anesthesia workload during teaching and nonteaching cases. Anesth Analg 2004:98:1419-25.

36 Weinger MB, Herndon OW, Zornow MH, Paulus MP, Gaba DM, Dallen LT. An objective methodology for task analysis and workload assessment in anesthesia providers. Anesthesiology 1994;80:77-92.

37 Borg G, ed. Simple rating methods of perceived exertion. Permagon Press, 1977.

38 Australian and New Zealand College of Anaesthetists. Recommendations on the recording of an episode of anesthesia care (the anaesthesia record) (Policy document Review PS 6). Australian and New Zealand College of Anaesthetists, 2001

39 Smedley BD, Stith AY, Nelson AR, eds. Unequal treatment-confronting racial and ethnic disparities in health care. National Academy Press, 2003.

40 Davis P, Lay-Yee R, Dyall L, Briant R, Sporle A, Brunt D, et al. Quality of hospital care for Māori patients in New Zealand: retrospective cross-sectional assessment. Lancet 2006:367:1920-5.

41 Currie M, Mackay P, Morgan C, Runciman WB, Russell WJ, Sellen A, et al. The "wrong drug" problem in anaesthesia: an analysis of 2000 incident reports. Anaesth Intensive Care 1993;21:596-601.

42 Zollinger RM Jr, Kreul JF, Schneider AJ. Man-made versus computer-generated anesthesia records. J Surg Res 1977;22:419-24.

43 Weinger MB, Herndon OW, Gaba DM. The effect of electronic record keeping and transesophageal echocardiography on task distribution, workload, and vigilance during cardiac anesthesia. Anesthesiology 1997;87:144-55.

44 Storr JA, Engineer C, Allan V. Save lives: clean your hands: a WHO patient safety initiative for 2009. World Hosp Health Serv 2009;45:23-5.

45 Orser BA, Oxorn DC. An anaesthetic drug error: minimizing the risk. Can J Anaesth 1994;41:120-4.

46 Parsons HM. What happened at Hawthorne? Science 1974:183:922-32.

47 De Amici D, Klersy C, Ramajoli F, Brustia L. More about the Hawthorne effect. Anesth Analg 2000;91:1043.

48 Evley R, Russell J, Mathew D, Hall R, Gemmell L, Mahajan RP. Confirming the drugs administered during anaesthesia: a feasibility study in the pilot National Health Service sites, UK. Br J Anaesth 2010;105:289-96.

Accepted: 9 August 2011

\section{Cite this as: BMJ 2011;343:d5543}

This is an open-access article distributed under the terms of the Creative Commons Attribution Non-commercial License, which permits use, distribution, and reproduction in any medium, provided the original work is properly cited, the use is non commercial and is otherwise in compliance with the license. See: http://creativecommons.org/licenses/by$\mathrm{nc} / 2.0 /$ and http://creativecommons.org/licenses/by-nc/2.0/legalcode. 


\section{Tables}

Table 1| Patients' characteristics, ASA score, duration of anaesthesia, case type (emergency/elective), and surgical specialty for participating patients in study comparing new system designed to reduce errors in recording and administration of drugs in anaesthesia and conventional methods. Figures are numbers (percentage) of patients unless otherwise specified

New system $(n=566)$ Conventional methods $(n=509)$

\begin{tabular}{|c|c|c|}
\hline Mean (SD) age years & $57(19)$ & $57(19)$ \\
\hline Men & $287(51)$ & $249(49)$ \\
\hline \multicolumn{3}{|l|}{ Ethnicity: } \\
\hline European & $370(65)$ & $322(63)$ \\
\hline Māori & $43(8)$ & $52(10)$ \\
\hline Pacific Island & $69(12)$ & $52(10)$ \\
\hline Other & $84(15)$ & $83(16)$ \\
\hline \multicolumn{3}{|l|}{ ASA score: } \\
\hline 1 & $137(24)$ & $112(22)$ \\
\hline 2 & $228(40)$ & $200(39)$ \\
\hline 3 & $170(30)$ & $169(33)$ \\
\hline 4 & $30(5)$ & $27(5)$ \\
\hline Not recorded & $1(0)$ & $1(0)$ \\
\hline Emergency case & $214(38)$ & $222(44)$ \\
\hline \multicolumn{3}{|c|}{ Duration of anaesthesia (hours): } \\
\hline$<1$ & $273(48)$ & $236(46)$ \\
\hline $1-2$ & $192(34)$ & $185(36)$ \\
\hline $2-3$ & $63(11)$ & $49(10)$ \\
\hline$\geq 3$ & $38(7)$ & $39(8)$ \\
\hline \multicolumn{3}{|l|}{ Surgical specialty: } \\
\hline General surgery & $175(31)$ & $174(34)$ \\
\hline Orthopaedic & $192(34)$ & $135(27)$ \\
\hline Urology & $159(28)$ & $171(34)$ \\
\hline Cardiothoracic & $36(6)$ & $28(6)$ \\
\hline Other & $4(1)$ & $1(0)$ \\
\hline
\end{tabular}

$\mathrm{ASA}=$ American Society of Anesthesiologists. 
Table 2| Error rates per 100 drug administrations for new system designed to reduce errors in recording and administration of drugs in anaesthesia (5680 administrations) and for conventional methods (5084 administrations)

\begin{tabular}{|c|c|c|c|}
\hline & New system ( $n=566$ cases) & Conventional methods ( $n=509$ cases) & $P$ value \\
\hline Overall error rate* $(95 \% \mathrm{Cl})$ & $9.1(6.9$ to 11.4$)$ & 11.6 (9.3 to 13.9$)$ & 0.045 \\
\hline \multicolumn{4}{|l|}{ Errors in drug administration: } \\
\hline Incorrect drug given (substitution) & 0.03 & 0.18 & 0.09 \\
\hline Drug not given (omission) & 0.11 & 0.14 & 0.63 \\
\hline \multicolumn{4}{|l|}{ Errors in drug recording: } \\
\hline Drug given, not recorded at all & 2.31 & 3.50 & 0.002 \\
\hline Drug given, dose not recorded & 0.05 & 0.67 & $<0.001$ \\
\hline $\begin{array}{l}\text { Discrepancy between recorded and observed } \\
\text { total dose }\end{array}$ & 6.86 & 7.18 & 0.94 \\
\hline Incorrect label† & 0.02 & 0.04 & 0.67 \\
\hline
\end{tabular}

*Derived from general linear mixed model, which included operating theatre as random factor, so might differ from error rate that can be derived from table 5. †Inconsistency between reconciled ampoule, labelled syringe, and drug recorded by anaesthetist. We were unable to identify in this situation whether intended drug was that recorded, given, or on label. 


\begin{tabular}{|c|c|c|}
\hline & Mean $(95 \% \mathrm{Cl})$ & $P$ value \\
\hline \multicolumn{3}{|l|}{ Ethnicity: } \\
\hline European & 10.2 (8.3 to 12$)$ & \multirow[t]{4}{*}{0.62} \\
\hline Māori & 11.6 (7.4 to 15.8$)$ & \\
\hline Pacific Island & 8.5 (4.7 to 12.3$)$ & \\
\hline Other & $11.3(8.0$ to 14.5$)$ & \\
\hline \multicolumn{3}{|l|}{ Sex: } \\
\hline Male & 11.7 (9.5 to 14.0$)$ & \multirow[t]{2}{*}{0.03} \\
\hline Female & 9.0 (6.7 to 11.3 ) & \\
\hline \multicolumn{3}{|l|}{ ASA score: } \\
\hline 1 & 9.2 (6.5 to 12.0$)$ & \multirow[t]{4}{*}{0.63} \\
\hline 2 & 11.0 (8.8 to 13.2 ) & \\
\hline 3 & 11.3 (8.9 to 13.7$)$ & \\
\hline 4 & $10.0(4.7$ to 15.2$)$ & \\
\hline \multicolumn{3}{|l|}{ Surgery type: } \\
\hline Emergency & $10.6(8.2$ to 13.0$)$ & \multirow[t]{2}{*}{0.78} \\
\hline Elective & 10.2 (8.0 to 12.4$)$ & \\
\hline
\end{tabular}

ASA=American Society of Anesthesiologists.

*Derived from general linear mixed model, which included operating theatre as random factor, so might differ from those that can be derived from table 5 . When included as a covariate, age was not significantly associated with error rate $(P=0.13)$. There was no significant difference between operating theatres $(P=0.4)$. 
Table 4| Mean (SE) times spent on various tasks in study comparing new system designed to reduce errors in recording and administration of drugs in anaesthesia with conventional methods

\begin{tabular}{|c|c|c|c|}
\hline Durations & New system ( $n=208$ cases) & Conventional methods ( $n=208$ cases) & P value ${ }^{\star}$ \\
\hline Drug preparation & $15 \mathrm{~min} 30 \mathrm{sec}(29 \mathrm{sec})$ & $14 \min 23 \mathrm{sec}(29 \mathrm{sec})$ & 0.06 \\
\hline Drug administration $†$ & $9 \min 2 \mathrm{sec}(18 \mathrm{sec})$ & $8 \mathrm{~min} 2 \mathrm{sec}(18 \mathrm{sec})$ & 0.01 \\
\hline $\begin{array}{l}\text { Establishing vascular access and establishing/adjusting } \\
\text { IV infusions }\end{array}$ & $10 \mathrm{~min} 36 \mathrm{sec}(30 \mathrm{sec})$ & $9 \min 36 \mathrm{sec}(30 \mathrm{sec})$ & 0.10 \\
\hline Airway procedures & $9 \min 45 \mathrm{sec}(38 \mathrm{sec})$ & $9 \min 43 \mathrm{sec}(39 \mathrm{sec})$ & 0.96 \\
\hline Adjusting anaesthetic equipment & $10 \min 12 \mathrm{sec}(33 \mathrm{sec})$ & $9 \min 47 \mathrm{sec}(33 \mathrm{sec})$ & 0.53 \\
\hline Positioning patient & $2 \min 42 \mathrm{sec}(9 \mathrm{sec})$ & $2 \min 29 \mathrm{sec}(9 \mathrm{sec})$ & 0.24 \\
\hline Observing monitors & $20 \min 22 \mathrm{sec}(63 \mathrm{sec})$ & $23 \min 1 \mathrm{sec}(64 \mathrm{sec})$ & 0.04 \\
\hline Observing patient & $28 \min 26 \mathrm{sec}(84 \mathrm{sec})$ & $21 \min 22 \mathrm{sec}(85 \mathrm{sec})$ & $<0.001$ \\
\hline Recording & $10 \min 23 \mathrm{sec}(41 \mathrm{sec})$ & $20 \min 5 \sec (42 \mathrm{sec})$ & $<0.001$ \\
\hline Tidying workspace & $3 \min 28 \mathrm{sec}(10 \mathrm{sec})$ & $3 \min 17 \mathrm{sec}(10 \mathrm{sec})$ & 0.33 \\
\hline Task and teaching related conversation & $33 \min 42 \mathrm{sec}(79 \mathrm{sec})$ & $31 \mathrm{~min} 27 \mathrm{sec}(80 \mathrm{sec})$ & 0.16 \\
\hline Task related reading & $1 \mathrm{~min} 41 \mathrm{sec}(12 \mathrm{sec})$ & $1 \mathrm{~min} 57 \mathrm{sec}(13 \mathrm{sec})$ & 0.30 \\
\hline Other tasks & $6 \min 10 \mathrm{sec}(31 \mathrm{sec})$ & $7 \mathrm{~min} 1 \mathrm{sec}(31 \mathrm{sec})$ & 0.17 \\
\hline
\end{tabular}

${ }^{*}$ Calculated by analysis of variance (ANOVA) with total task duration and theatre as covariates in general linear mixed model.

†Drug administration included administering IV (intravenous) drugs by bolus and infusion and adjusting vapourisers and gas flow. 
Table 5 Specific drug administration errors and incorrect label errors in study comparing new system designed to reduce errors in recording and administration of drugs in anaesthesia with conventional methods. Number of times each event occurred is given in brackets

\begin{tabular}{|c|c|c|}
\hline Error type (type total) & New system & Conventional methods \\
\hline \multicolumn{3}{|l|}{ Substitution* (9): } \\
\hline Interclass (1) & - & Glycopyrrolate for atracurium (1) \\
\hline Intraclass (8) & $\begin{array}{l}\text { Atracurium for vecuronium (1); remifentanil for alfentanil } \\
\text { (1) }\end{array}$ & $\begin{array}{l}\text { Cefoxitin for cefuroxime (1); cephazolin for cefoxitin (3); } \\
\text { cephazolin for cefuroxime (1); glycopyrrolate for atropine (1) }\end{array}$ \\
\hline Omission (13) & $\begin{array}{l}\text { Antibiotic }(1) \text {; atropine (2); gentamicin (1); heparin (1); } \\
\text { morphine (1); salbutamol (1) }\end{array}$ & $\begin{array}{l}\text { Dexamethasone (1); droperidol (1); metaraminol (2); morphine } \\
\text { (1); vecuronium (1) }\end{array}$ \\
\hline Incorrect label (5) & $\begin{array}{l}\text { Atracurium labelled vecuronium (1); atracurium labelled } \\
\text { rocuronium (1) }\end{array}$ & Glycopyrrolate labelled atropine (2); incorrect dilution (1) \\
\hline Total & 11 & 16 \\
\hline
\end{tabular}

*Substitution errors between (interclass) and within (intraclass) pharmacological classes as defined by international colour code for anaesthetic drugs. †No antibiotics given. 


\section{Figure}

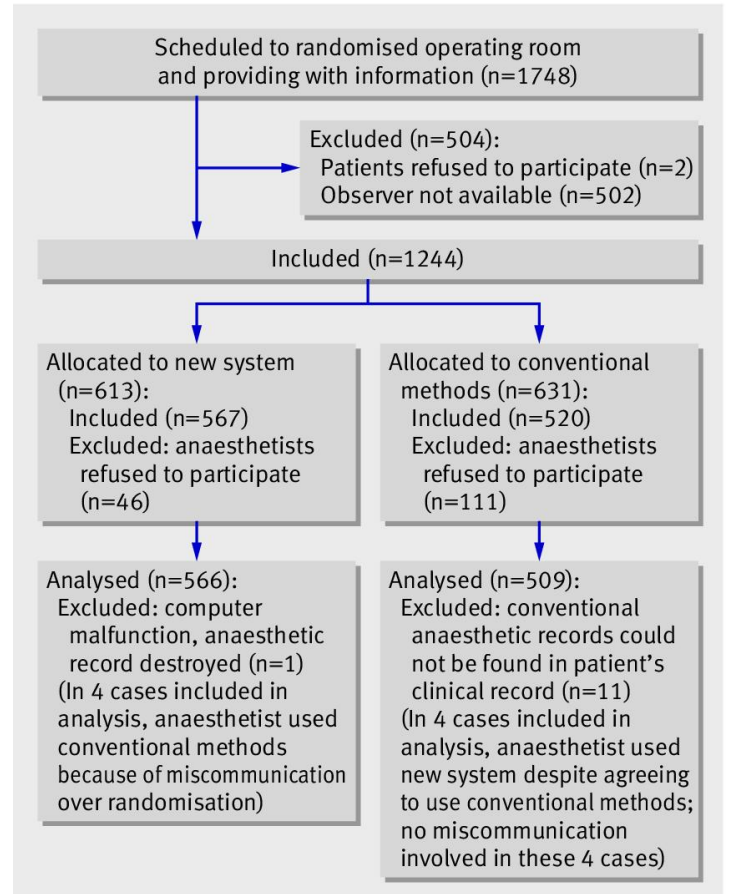

Flow of cases (patients undergoing anaesthesia for surgery) through trial; five operating theatres randomly allocated by week to one or other method for 83 study weeks 